\title{
Markov Chain Model to Explain the Dynamics of Human Depression
}

\author{
Souvik Bhattacharya \\ Department of Mathematics, University of Florida, 358 Little Hall, P.O. Box 118105, Gainesville, FL 32611-8105, USA \\ Correspondence should be addressed to Souvik Bhattacharya; souvik@ufl.edu
}

Received 25 August 2013; Revised 3 February 2014; Accepted 11 February 2014; Published 18 March 2014

Academic Editor: Mitsuhiro Ohta

Copyright (C) 2014 Souvik Bhattacharya. This is an open access article distributed under the Creative Commons Attribution License, which permits unrestricted use, distribution, and reproduction in any medium, provided the original work is properly cited.

\begin{abstract}
Depression is one of the major concerns of the present generation. A Markov chain model has been used to portray and investigate this curse. Long-term behaviour of the model has been discussed. Different types of treatment strategies have been considered in this paper to identify the most powerful measure of keeping this disease from its spread in the society. This paper also focuses on the usefulness of the drugs available at present for the treatment of this disease.
\end{abstract}

\section{Introduction}

Depression is one of the major diseases which is affecting the present generation. The fast-paced life has posed its own curse on the society. The tensions that come as a supplement to the six-figure salary is unavoidable. This disease is associated with lowered work functioning, including absences, impaired productivity, and decreased job retention. Several manuscripts have been published on this issue to find all possible ways to eliminate this from the society. In one of the articles, [1] the author introduces a Markov chain model which provides a method to deal with various sequence of information. The most common form of depression, known as Major Depressive Disorder (MDD), is modeled and analyzed by the Markov chain model. The author successfully obtained a mean to find the future state of the depression based on the present situation. Some manuscripts deal with the numerical computations on moods and depression [2]. In other articles [3], the author has explained the psychological theories about a unipolar clinical depression through proper representation using a well-designed mathematical model. In this paper, the author took great interest in captivating the dynamics of mood in human or human-like agents. The articles listed above clearly portray the idea that there is a great concern of controlling depression in the present generation. Most of the models could successfully capture the salient features of a person's state, but it is rather a hard task to explain the long-term behaviour of a person's state.
The daily commotions create disturbances in an individual's life, which is sufficient in creating a disturbance in his normal state of mind. The effectiveness of these disturbances would determine the probability of drifting an individual towards a depressed state. In order to study the causes behind this depression, the scientists are also taking into concern the dopaminergic and serotonergic synapses in an individual $[4,5]$. Experimental studies are also carried out to control this disease. In [6], the author developed a screening instrument for depression using items from the Minimum Data Set of the Resident Assessment Instrument. Cortical depression has been analysed too in [7]. Depression Anxiety Stress Scales (DASS) are one of the well-known scales of measuring the degree of depression. Several articles have been published using this measuring scale [8-10].

In our present model, we have analysed a two-state Markov chain model. In order to avoid the complexity involved in mathematical calculations, we have only restricted to a two-state model. This model has been used to identify the long-term behaviour of the depressed state. We have used both mathematical analysis and numerical simulation to validate the argument presented in the text.

\section{Two-State Markov Chain Model}

Depression is one of the major villains of this society. The fast-paced life of the present generation has created several 


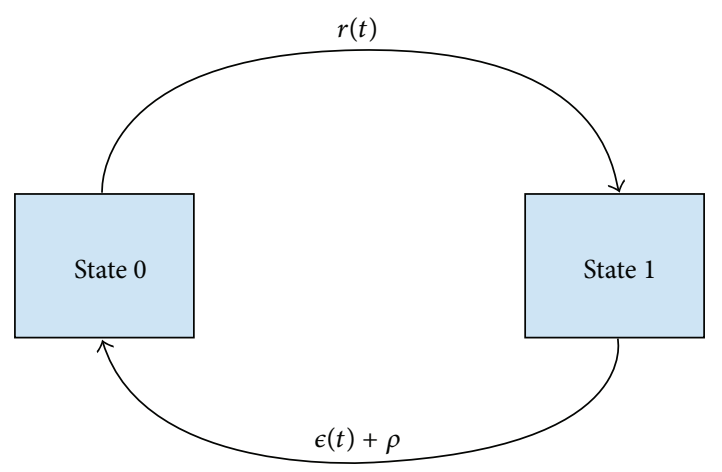

FIgure 1: Two-state Markov chain model.

problems in their lives. The rush hours in the metropolitan cities could identify thousands of individuals suffering from this disease. It is $7 \mathrm{a} . \mathrm{m}$. in the morning and one has to be in his workplace by 7:30 a.m. It creates anxiety within oneself. This anxiety would subside as the phase gets over. But if this persists for long, it creates disturbances in the chemical agents present in our brain, which affects our health. It is absolutely perfect to feel sad or nervous sometimes, but there should be some reason behind it. Normal tensions and worries stay for a day or two. If the phase tends to last longer, then it is necessary to take advice from an expert like a psychiatrist or a psychologist. These experts are trained to identify the problem and assist their clients in solving it. There is welldesigned questionnaire, which has been carefully structured to assess the major cause of depression within the patient $[11,12]$. When people feel blue or worried with a good cause, it does not demolish their ability of social behaviour, but when depression takes its role, it would bring every possible hurdle in one's life and workplace. It is probably the best time to visit a psychiatrist. There are several existing models, to monitor a patients' state and behaviour with environmental sensors [1315]. In our present model, we have used a continuous time Markov chain transitional probability model, to describe the depressive behaviour in patients. We begin our model with the simplest case. In this paper, we have explored a two-state Markov chain model. The two states are state- 0 and state- 1 , where the former represents the state of a normal person who does not show any identifiable depressive disorder within himself and the second state represents the state where the person can be categorized as one suffering from depression as per the scales defined by the psychiatrist. Figure 1 shows the transitional behaviour of depression.

This model is different from the existing models in the way that it assumes that the transitional probabilities are not constant. The different hassles of life drive a normal person from state- 0 to state- 1 . The function $r(t)$ represents the rate of transition from the state of a normal human being to the state of a depressed individual. The function $r(t)$ varies from person to person and on the external effect of social life on the individual. We begin our investigation assuming that $r(t)=r_{0}$, a constant, which would signify that there is a constant external pressure on the individual which would eventually drag him towards depressive state.
We would also explore the case, when $r(t)$ would be a periodic function, so as to capture the scenario, when the pressure builds up on an individual not at a constant rate, but it is periodic in nature. The pressure, which is responsible for dragging an individual towards depressive state, repeats itself after a certain interval. When a person moves to the depressive state, the prime requirement for the doctor is to bestow a normal life to him. One of the major treatments the experts try on their patients is consultation, where the doctor verbally tries to kindle the positive thoughts within the individual and change his state to that of a normal person. This is assumed as $\rho$ in this model. In the following sections, two different forms of $\rho$ have been explored and discussed to excavate the difference in the two forms and evaluate which form is better for the patient. An article published in the journal Science dated September 17, 2010, shows that the drug "Prozac" is effective in increasing the serotonin content of the brain. With the invention of several chemical drugs, the psychiatrists also recommend their patients to take these pills, so as to improve their condition. Since the pills play a chemical reaction to increase the level of serotonin in the brain, the impact is not permanent and hence the psychiatrists recommend consuming these pills periodically. Hence, the effect of pills in recovery from state 1 to state 0 has been introduced in this model through $\epsilon(t)$. Since this only creates a periodic improvement in the life of an individual, $\epsilon(t)$ has been assumed to be a periodic function of time. In this paper, a continuous time Markov chain model has been used to represent the changes in the probabilities in each state. Let $p_{0}(t)$ represent the probability of being in the state0 and let $p_{1}(t)$ map the probability of state- 1 . The following differential equation model represents the entire picture:

$$
\begin{gathered}
p_{0}^{\prime}(t)=-r(t) p_{0}(t)+(\rho+\epsilon(t)) p_{1}(t), \\
p_{1}^{\prime}(t)=r(t) p_{0}(t)-(\rho+\epsilon(t)) p_{1}(t) .
\end{gathered}
$$

Note that the above-presented system is a closed ODE and $p_{0}+p_{1}=1$. Hence, it can be reduced to an ordinary differential equation in one variable. Consider

$$
p_{1}^{\prime}=-(\rho+\epsilon(t)+r(t)) p_{1}+r(t) .
$$

In the following section, we explore the various features of $p_{1}$ under different situations.

\section{Analysis of the Two-State Markov Chain Model}

The model presented above can be integrated to obtain the solution of the probability in state- 1 as

$$
p_{1}(t)=p_{1}(0) e^{-\int_{0}^{t}(\rho+\epsilon(s)+r(s)) d s}+\int_{0}^{t} r(s) e^{-\int_{s}^{t}(\rho+\epsilon(u)+r(u)) d u} .
$$

The behaviour of the model (2) under different conditions will now be explored. 


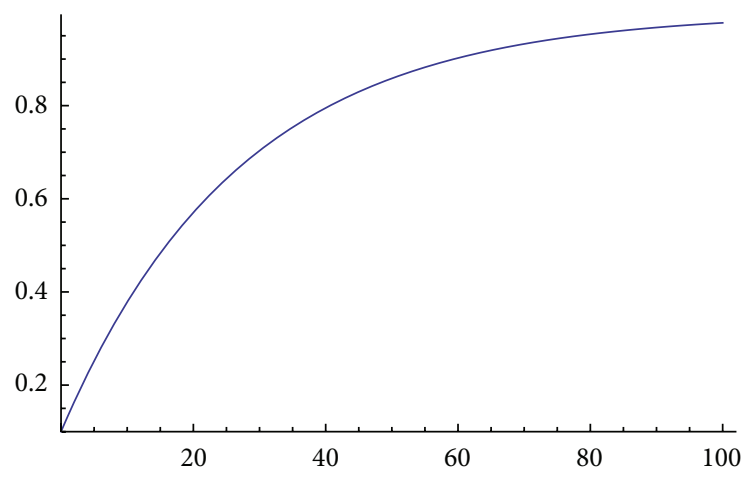

Figure 2: Behaviour of $p_{1}$ in the absence of treatment.

3.1. $\rho=0, \epsilon=0$, and $r(t)=r_{0}$ Is a Constant. We begin our analysis with the simplest assumption that there is no treatment for the patient either through medication or personal interaction with the consultants. The source of depression is assumed to be constant $r_{0}$. With the above-mentioned restriction, we obtain the following ODE:

$$
p_{1}^{\prime}=-r_{0} p_{1}+r_{0}
$$

which can be integrated to obtain the following solution:

$$
p_{1}=\left(p_{1}(0)-1\right) e^{-r_{0} t}+1=\left(1-e^{-r_{0} t}\right)+p_{1}(0) e^{-r_{0} t},
$$

as $t \rightarrow \infty$; we can see that $p_{1} \rightarrow 1$, which is an expected scene when there is no treatment available for the patient. The probability of state- 1 increases to 1 and the patient approaches to the depressed state with probability 1.

Figure 2 depicts this situation.

Figure 2 shows that $p_{1}(t)$ increases to 1 as $t \rightarrow \infty$, when there is no treatment available for the patient. Mathematica 9.0 has been used to draw the curve. The parameter used in this graph is $r_{0}=0.037$.

3.2. $\rho=\rho_{1}$ and $r(t)=r_{0}$ Are Constant and $\epsilon=0$. In this section, we will discuss the situation when the patient is under treatment. The form of treatment is discussed in this category and also in the section following it is only restricted to verbal consultation. The rate is constant here, which means that the patient receives a constant rate of consultation. We are assuming that there is no medication involved in treatment under this category. With this assumption, the equation described in (2) takes the form

$$
p_{1}^{\prime}=-\left(\rho_{1}+r_{0}\right) p_{1}+r_{0} .
$$

Integrating the above equation, we obtain the following solution for the probability:

$$
p_{1}=p_{1}(0) e^{-\left(\rho_{1}+r_{0}\right) t}+\frac{r_{0}}{\rho_{1}+r_{0}}\left[1-e^{-\left(\rho_{1}+r_{0}\right) t}\right] .
$$

As $t \rightarrow \infty$, the probability of state- 1 , that is, $p_{1}$, approaches $r_{0} /\left(\rho_{1}+r_{0}\right)$, which is less than one. Hence, this proves that consultation is effective in bringing down the probability of depressed state.

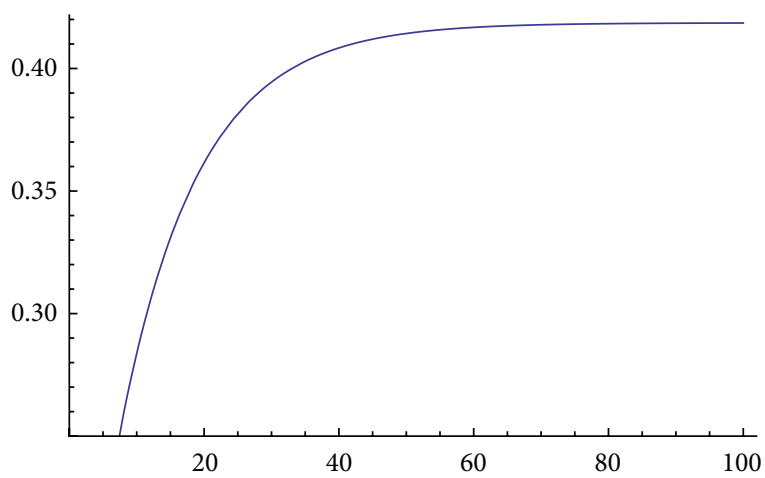

FIgURE 3: Behaviour of $p_{1}$ in the presence of treatment through consultation, when the rate is constant.

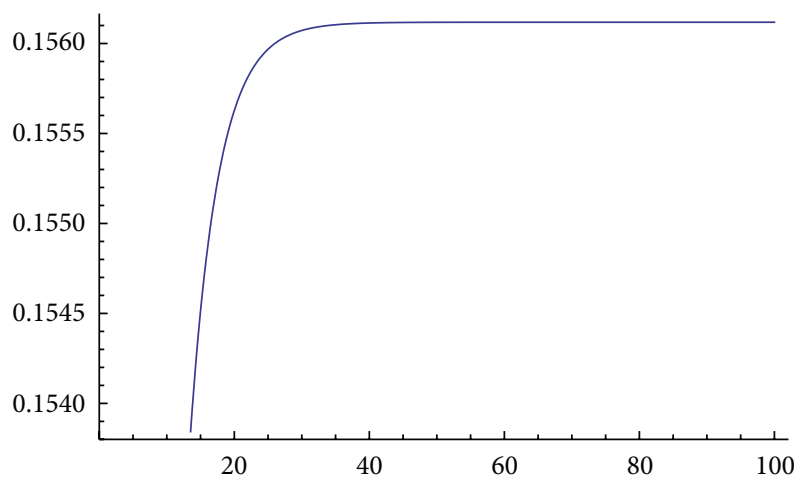

FIgURE 4: Behaviour of $p_{1}$ in the presence of treatment through consultation, when the rate is constant.

Figure 3 can be used as a reference to explain this scenario.

Parameters used in this graph are $r_{0}=0.037$ and $\rho_{1}=$ 0.05 . The graph clearly shows that the equilibrium value when it stabilizes is less than one, proving the fact that consultation is an effective measure in the treatment of depression. More consultation means higher value of $\rho_{1}$ and hence it leads in lowering the steady state probability of state-1. Figure 4 explains the case explicitly.

Parameters used in this graph are $r=0.037$ and $\rho_{1}=0.2$. The treatment rate has been increased from 0.05 to 0.2 , which brings an effective decline in the stabilizing value of $p_{1}(t)$; that is, the probability of depressed state gets lowered asymptotically, but it does not decay to 0 . Lowering the transition to depressed state would demand more consultation that is, increasing the rate of treatment. It is observed that using the same treatment rate only leads to lowering the state of depression but does not eradicate it completely. The following paragraph discusses a method which shows that mere changing the method of consultation can lead in achieving major results.

Let us define a function

$$
f(t)= \begin{cases}\rho_{1} t & \text { if } t \leq N, \\ \rho_{2} & \text { if } t>N,\end{cases}
$$


where $\rho_{1}$ is a constant such that $0<\rho_{1}<1 / N$ and the other constant $0<\rho_{2}<1$.

3.2.1. $\rho=f(n), \epsilon=0$, and $r(t)=r_{0}$. Here, we will analyse a situation, where the treatment strategy is somewhat more modified than the previous state. Instead of assuming a constant treatment through consultation, we would like to observe what would happen if the treatment rate is increased every time the patient visits the consultant. This would mean increasing the length of consultation or using improved techniques to boost the patient's mental state. We will conduct a mathematical treatment to explore the effectiveness of this strategy over the previous one. Note that both probabilities in the function $f(n)$ are less than one and, hence, the transitional probabilities are well defined. With the above-mentioned restrictions on the parameter, the differential equation takes the following form in the interval $0<t \leq N$ :

$$
p_{1}^{\prime}=-\left(\rho_{1} t+r_{0}\right) p_{1}+r_{0} .
$$

The solution would be of the form

$$
p_{1}=p_{1}(0) e^{-\left(\rho_{1} t^{2} / 2+r_{0} t\right)}+r_{0} e^{-\left(\rho_{1} t^{2} / 2+r_{0} t\right)} \int_{0}^{t} e^{\left(\rho_{1} s^{2} / 2+r_{0} s\right)} d s .
$$

This solution can be expressed in the form

$$
\begin{aligned}
p_{1}= & p_{1}(0) e^{-\left(\rho_{1} t^{2} / 2+r_{0} t\right)} \\
& +r_{0} e^{-\left(\rho_{1} / 2\right)\left(t+r_{0} / \rho_{1}\right)^{2}} \int_{0}^{t} e^{\left(\rho_{1} / 2\right)\left(s+r_{0} / \rho_{1}\right)^{2}} d s .
\end{aligned}
$$

Using the transformation $x=\sqrt{\rho_{1} / 2}\left(t+r_{0} / \rho_{1}\right)$, we can rewrite (11) to the form as

$$
\begin{aligned}
p_{1}(t)= & p_{1}(0) e^{-\left(\rho_{1} t^{2} / 2+r_{0} t\right)}+r_{0} e^{-x^{2}} \int_{r_{0} / \sqrt{2 \rho_{1}}}^{x} e^{s^{2}} d s \\
p_{1}(x)= & p_{1}(0) e^{-x^{2}-r_{0}^{2} / 2 \rho_{1}}+r_{0} e^{-x^{2}} \int_{0}^{x} e^{s^{2}} d s \\
& -r_{0} e^{-x^{2}} \int_{0}^{r_{0} / \sqrt{2 \rho_{1}}} e^{s^{2}} d s \\
= & I_{1}+I_{2}-I_{3} .
\end{aligned}
$$

The first and the last term, namely, $I_{1}$ and $I_{3}$, are bounded by $e^{-x^{2}}$. Since we have used the transformation $x=\sqrt{\rho_{1} / 2}(t+$ $\left.r_{0} / \rho_{1}\right)=\sqrt{\rho_{1} / 2} t+r_{0} / \sqrt{2 \rho_{1}}>r_{0} \sqrt{N} / \sqrt{2}$ as $t \rightarrow \infty$ and $N>t$, we have $N \rightarrow \infty$ which leads to $x \rightarrow \infty$. Hence, the integrals $I_{1}$ and $I_{2}$ can be made as small as possible. To show the boundedness of $I_{2}$, we use the following theorem.

Theorem 1. Let $I_{2}=r_{0} e^{-x^{2}} \int_{0}^{x} e^{s^{2}} d s$. As $x \rightarrow \infty, I_{2}$ can be made as small as possible.

Proof. At the very first stage, we observe that the integral, $I_{2}$, is a Dawson's integral $D_{+}(x)$. Obtaining the convergence of this integral is the same as finding the bound of the Dawson's integral. The function $I_{2}$ can be expressed in the following form:

$$
I_{2}=\frac{r_{0} e^{-x^{2}}}{2 \sqrt{\pi}} \int_{-\infty}^{\infty} g(t) d t,
$$

where $g(t)=e^{-t^{2}} t^{-1} \sinh (2 x t)$. Detail of the proof is given in [16]. Using the recipe mentioned in the manuscript [16], the integral of $g(t)$ can be rewritten using the rule of quadrature of step size $h$,

$$
\int_{-\infty}^{\infty} g(t) d t=Q(g ; h, \alpha)+E(g ; h, \alpha),
$$

where the quadrature sum is given by $Q(g ; h, \alpha)=$ $h \sum_{n=-\infty}^{\infty} g(n h+\alpha h), \alpha \in[0,1)$ is the shift which remains at our disposal, and $E(g ; h, \alpha)$ denotes the quadrature error. Following the computations exactly in the similar way explained in [16], we can obtain the following approximation for $I_{2}$ :

$$
I_{2}=r_{0} \pi^{-1 / 2} e^{-x^{2}}\left[2 h x+\sum_{\text {neven }} n^{-1} e^{-(x-n h)^{2}}\right]+r_{0} R(g ; 2 h, 0),
$$

where $R(g ; 2 h, 0) \leq 2 h r_{0} e^{-\pi^{2} / 4 h^{2}} / \pi\left(1-e^{-\pi^{2} / 4 h^{2}}\right) \approx$ $2 r_{0} h \pi^{-1} e^{-\pi^{2} / 4 h^{2}}$ gives the bound of the error term.

Note that $\sum_{\text {neven }} n^{-1} e^{-(x-n h)^{2}}$ is a finite sum and hence the term inside bracket in (15) can be bounded by a suitable constant. This shows that the first term in the expression of $I_{2}$ in (15) can be made as small as possible. The bound of the second term follows with smaller values of step size $h$. Thus, the function $I_{2}$ can be made as small as possible for large values of $x$. This completes the proof.

The theorem presented above proves that changing the strategy of consultation might result in drifting a patient completely from a depressed state to a completely normal human being. If the duration of treatment is increased, the probability of driving towards the depressed state can be reduced to a great extent. With the same treatment rate, if the method is modified, that is, keeping the contact hours of consultation the same, if the patient ispresented with better techniques of treatment every time he visits the doctor, then it would be completely effective in shifting the patient completely to the state of a normal human being.

But the relapse phase occurs when this treatment is stopped. In real life, it is impossible to assume infinite treatment. In this section, we will explore the situation, when this treatment phase is over and $\rho=\rho_{2}, t>N$; that is, the treatment is either reduced to regular minimised consultation or nothing. Hence, for $t>N$, we have the following differential equation:

$$
p_{1}^{\prime}=-\left(\rho_{2}+r_{0}\right) p_{1}+r_{0},
$$

which can be solved to obtain the following solution:

$$
p_{1}=p_{1}(N) e^{-\left(\rho_{2}+r_{0}\right)(t-N)}+\frac{r_{0}}{\rho_{2}+r_{0}}\left[1-e^{-\left(\rho_{2}+r_{0}\right)(t-N)}\right] .
$$




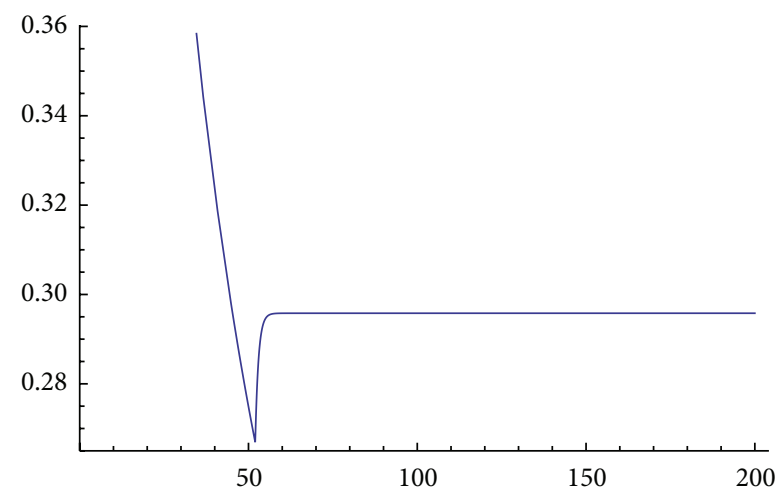

Figure 5: Behaviour of $p_{1}$ in the presence of treatment through consultation, when the rate is $\rho$ as defined in this section. The parameter values used here are $r_{0}=0.31, \rho_{1}=0.0167, \rho_{2}=0.738$, $N=52$, and $p_{1}(0)=0.5$.

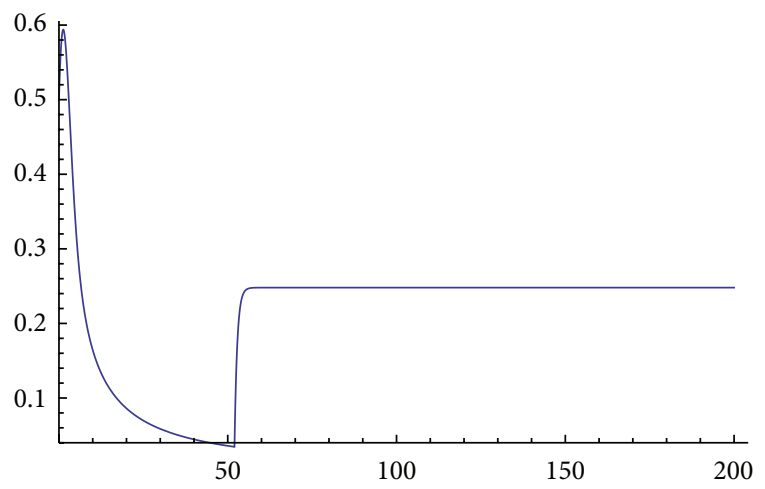

Figure 6: Behaviour of $p_{1}$ in the presence of treatment through consultation, when the rate is $\rho$ as defined in this section. The parameter values used here are $r_{0}=0.31, \rho_{1}=0.0167, \rho_{2}=0.94$, $N=52$, and $p_{1}(0)=0.5$.

Since $p(N)$ is small and when $t$ is close to $N$, we have $t-N \sim 0$ and, hence, the overall probability is low. But when $t$ drifts away from $N$, the term $e^{-\left(\rho_{2}+r_{0}\right)(t-N)}$ gets smaller and the resultant probability approaches a fixed constant $r_{0} /\left(\rho_{2}+r_{0}\right)$. The only possibility to restrain this phase of depression is to reduce $r_{0}$, which means either the outside pressure which causes depression reduces or it develops some inner strength within the individual, which is capable of fighting against the outside pressure, that drives towards depression.

Note that both the graphs show similar behaviour in this case as discussed in the section. Here, we have chosen $N=$ 50, $\rho_{1}=0.167<1 / 50$. During the treatment phase with this improved technique, it shows a steady decrease in the transitional probability towards depressed state. But once it stops, $r_{0}$ drives the individual towards depressed state. With an effective $\rho_{2}$, it merely reduces the stabilizing value, which is evident from Figures 5 and 6.

If $r_{0}$ is reduced, keeping the same $\rho_{2}$, it is found that the stabilizing value is reduced to a greater extent. If the new value of $r_{0}$ is set at 0.07 , Figure 7 shows that the stabilizing value is much lowered. Hence, if the treatment strategy is able

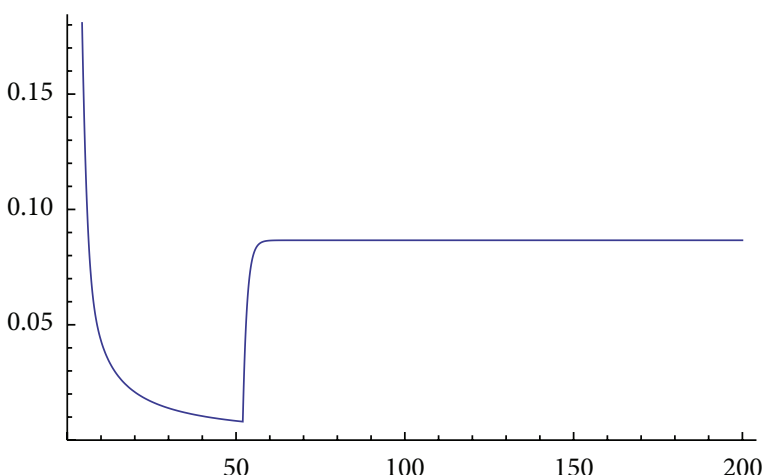

FIgURE 7: Behaviour of $p_{1}$ in the presence of treatment through consultation, when the rate is $\rho$ as defined in this section. The parameter values used here are $r_{0}=0.07, \rho_{1}=0.0167, \rho_{2}=0.738$, $N=52$, and $p_{1}(0)=0.5$.

to develop the positive feeling among the individual to fight against depression, it is possible to reduce the stabilizing state.

In the later part of this paper, we will discuss cases when $r(t)$ is not a constant, that is, the rate of driving a person into depressed state is not just a constant, and also we will see the effect of medicine in curing a depressed patient.

3.3. $\epsilon=0, r(t)$ Is a Nonconstant Function, and $\rho=\rho_{1}$ Is Constant. In practical life, the tensions and other causes of depression are not a constant function. It changes with time. Since the phase of depression repeats itself with the daily tensions from various sources and again it subsides when the phase is over, we can model $r(t)$ with an almost periodic function. In this section, we will explore the effect of the depression when the treatment is at a constant rate.

Before we begin our analysis, we put forth the definition of an almost periodic function.

Definition 2. An almost periodic function is a function defined on the set of real numbers that is periodic to within any desired level of accuracy. It is a function whose value is approximately repeated when its argument is increased by properly selected constants (the almost periods).

This reduces the ODE (2) presented in this section to the following form:

$$
p_{1}^{\prime}=-(\rho+r(t)) p_{1}+r(t)
$$

We can rewrite the equation in the form

$$
\begin{gathered}
p_{1}^{\prime}+(\rho+r(t)) p_{1}-r(t)=0, \\
p_{1}^{\prime}(t)+g\left(p_{1}, t\right)=0,
\end{gathered}
$$

where $g\left(t, p_{1}\right)=(\rho+r(t)) p_{1}-r(t)$ is an almost periodic function of $t$.

We use the following theorems presented in [17] to prove the unique existence of almost periodic solutions in (19).

Theorem 3. Assume that $g=g\left(t, p_{1}\right): \mathbb{R} \times \mathbb{R} \rightarrow \mathbb{R}$ is nondecreasing wrt $p_{1}$ and almost periodic of $t$, uniformly wrt 


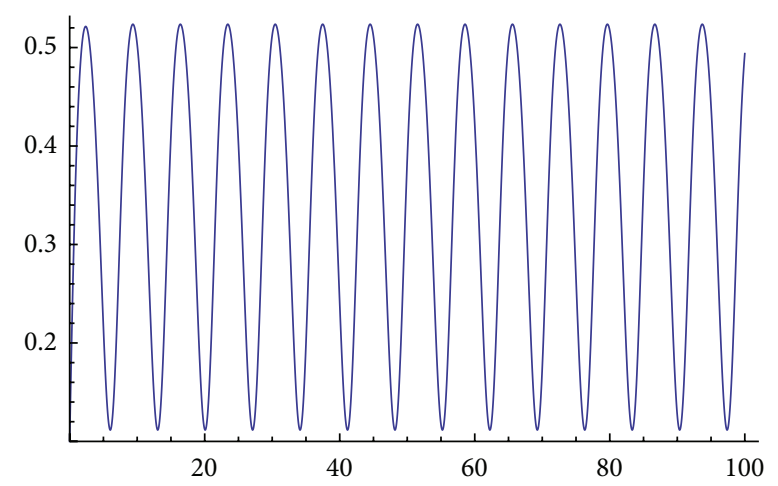

Figure 8: Behaviour of $p_{1}$ in the presence of treatment through consultation, when the rate is constant but $r(t)$ is periodic function.

$p_{1}$ on bounded sets. If there is $M>0$ such that $g(t ;-M) \leq 0 \leq$ $g(t ; M)$, for all $t \in \mathbb{R}$, then there is at least one almost periodic solution $p_{1}$ for (19) satisfying $-M \leq p_{1}(t) \leq M, \forall t \in \mathbb{R}$.

Since $g$ is both nondecreasing wrt $p_{1}$ and periodic wrt $t$, the above theorem applies well in this case, which guarantee the existence of an almost periodic solution. The paper also proved the uniqueness of the periodic solution. This shows that, with the daily commotions, it is not possible to bring down the depressed state with constant $\rho$. Figure 8 shows a numerical simulation of this system.

We have used a periodic function $r(t)=0.5(\sin (0.894 t)+$ 1 ) and the value of $\rho_{1}$ has been adjusted at 0.85 . The graph clearly shows the fact that even with regular consultation when the rate of consultation is constant, oscillations persist in the system. Increasing the rate of consultation would merely reduce the range of oscillation, but it will not eradicate the possibility of getting depressed completely.

We again define the function

$$
f(t)= \begin{cases}\rho_{1} t & \text { if } t \leq N \\ \rho_{2} & \text { if } t>N\end{cases}
$$

where $\rho_{1}$ is a constant such that $\rho_{1}<1 / N$.

3.4. $\epsilon=0, r(t)$ Is a Nonconstant Function, and $\rho=f(t)$. In this section, we would explore the same model presented above, when we use the modified consultation strategy. Here, $\rho=\rho_{1} t$, where $\rho_{1}$ is a constant. This reduces the ODE to the following form:

$$
p_{1}^{\prime}=-(\rho t+r(t)) p_{1}+r(t)
$$

when $t<N$.

Note that (22) can be bounded by the following function:

$$
p_{1}^{\prime} \leq-\rho t p_{1}+r_{0}
$$

where $r_{0}=\sup _{t>0} r(t)$ gives an upper bound of the rate of depression. Solving (23), we obtain the following bound on the solution of $p_{1}(t)$ :

$$
p_{1} \leq p_{1}(0) e^{-\rho t^{2} / 2}+r_{0} e^{-\rho t^{2} / 2} \int_{0}^{t} e^{\rho s^{2} / 2} d s .
$$

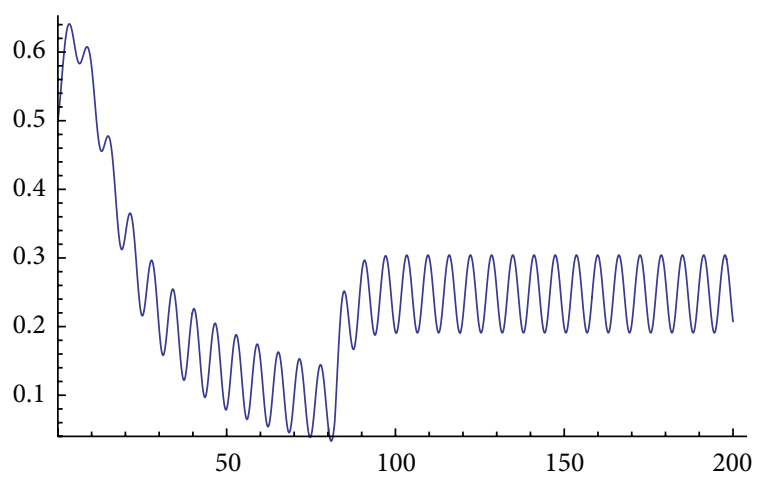

FIGURE 9: Behaviour of $p_{1}$ in the presence of treatment through consultation, when the rate is nonconstant but $r(t)$ is periodic function.

The 1st term in the equation can be made as small as possible by taking large $N$. The second term is a Dawson's Integral and we have proved in the earlier section that this integral can be made as small as possible. Hence, this proves that $p_{1}$ can be made as small as possible if $\rho(t)=\rho_{1} t$ for large $N$.

For the time period $t>N$, we have

$$
p_{1}^{\prime}=-\left(\rho_{2}+r(t)\right) p_{1}+r(t) .
$$

Using similar argument presented in Section 3.3, we can show that the probability $p_{1}$ persists with a sustained oscillation. Figure 9 explain this situation clearly.

We have used a periodic function $r(t)=0.079(\sin (t)+1)$ and the values of $\rho_{1}=0.01, N=82$, and $\rho_{2}=0.236$. Note here $\rho_{1}=0.01<1 / N=1 / 82$ which satisfies the criterion of the transitional probability explained in this section. Here, the initial value is $p_{1}(0)=0.5$. The stabilizing frequency is between 0.2 and 0.25 .

3.5. $\epsilon$ Is a Periodic Function, $r(t)$ Is a Nonconstant Function, and $\rho=\rho_{1}$ Is a Constant Term. Now, we will discuss the general situation when $\epsilon(t)$ is a general function. $\epsilon(t)$ represents the treatment through medications. It is believed that these drugs improve the symptoms of depression by increasing the availability of certain brain chemicals called neurotransmitters. It increases slowly following the pharmacodynamic nature of medicine. Hence, we will assume it to be periodic in nature. With the assumption that $r(t)$ is an almost periodic function, we can rewrite the ODE as $p_{1}^{\prime}+C\left(t, p_{1}\right)=0$, where $C\left(t, p_{1}\right)=\left(r(t)+\rho_{1}+\epsilon(t)\right) p_{1}+r(t)$ is an almost periodic function. From the theorem stated above, we can conclude that there exists an almost periodic solution for this equation, which is unique. Hence, even in this case, oscillations persist. Figure 10 proves this fact.

We have used a periodic function $r(t)=0.155(\sin (t)+1)$ and the value of $\rho=\rho_{1}=0.538$. We have used a periodic function to represent $\epsilon(t)=0.15(1+\sin (t))$. The initial value of $p_{1}(0)$ has been assumed to be 0.5 . This proves that although medication can bring down the chance of depression initially, it cannot completely remove it and, hence, medications need to be repeated for a depressed patient forever. 


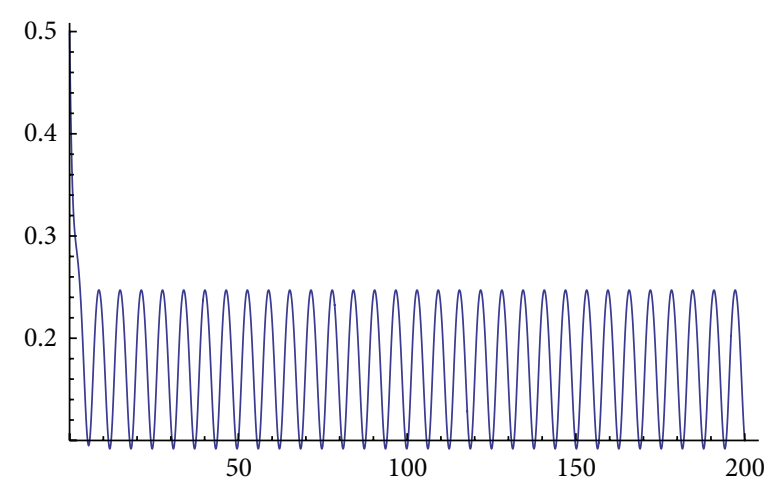

Figure 10: Behaviour of $p_{1}$ in the presence of treatment through consultation, when the rate is nonconstant but $r(t)$ is periodic function.

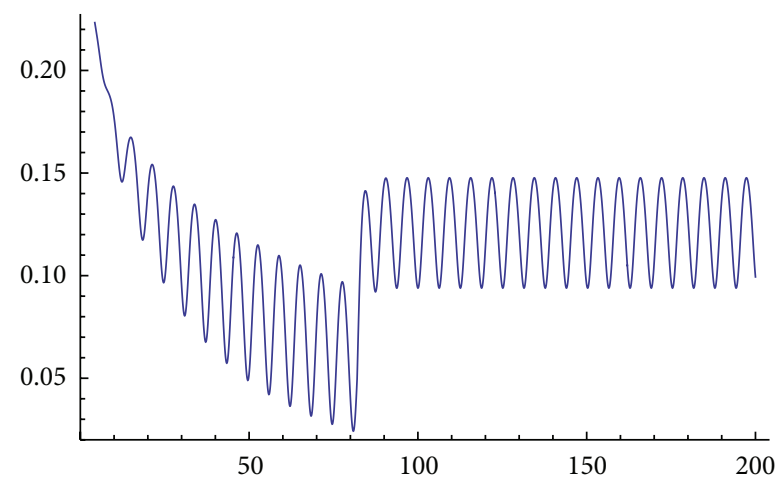

FIGURE 11: Behaviour of $p_{1}$ in the presence of treatment through consultation, when the rate is nonconstant but $r(t)$ is periodic function.

3.6. $\epsilon$ Is a periodic function, $r(t)$ Is a Nonconstant Function, and $\rho=\rho_{1} t$. In this section, we will finally conclude that, even with the use of drugs, if $\rho=f(t)$ in the same way defined before,

$$
f(t)= \begin{cases}\rho_{1} t & \text { if } t \leq N \\ \rho_{2} & \text { if } t>N\end{cases}
$$

Since $\epsilon$ is a bounded being a periodic function, the bounds of $\epsilon$ are so chosen such that $\max (\epsilon)<1-\max \left[\rho_{2}, \rho_{1} *\right.$ $N]$. Let us consider the function when $t \leq N$.

The ODE presented above in (2) can be bounded by a converging ODE

$$
p_{1}^{\prime}=-\left(\rho_{1} t+\epsilon(t)+r(t)\right) p_{1}+r(t) \leq-\left(\rho_{1} t+\epsilon(t)\right) p_{1}+r_{0},
$$

where $r_{0}=\sup _{t>0} r(t)$. By the argument presented above, we can observe that $p_{1}$ can be made arbitrarily small as $t \rightarrow \infty$. Hence, a change in consultation can bring a great reduction in depression in a patient. For time period when $t>N$, the equation reduces to the form

$$
p_{1}^{\prime}=-\left(\rho_{2}+\epsilon(t)+r(t)\right) p_{1}+r(t)
$$

Since both the terms, $\epsilon(t)$ and $r(t)$ are periodic function, this equation is similar to the form $p_{1}^{\prime}+C\left(t, p_{1}\right)=0$, where $C\left(t, p_{1}\right)=\left(r(t)+\rho_{2}+\epsilon(t)\right) p_{1}+r(t)$ is an almost periodic function. By the argument presented in the above theorem, we can claim that there exists a periodic solution to the ODE in (28).

Figure 11 assumes that the probability of depression starts from $p_{1}(0)=0.5$ and the parameters used are $r(t)=0.079(1+$ $\sin (t)), \rho_{1}=0.01, \rho_{2}=0.236$, and $N=82$. Note that these parameters are exactly the same as the graph in Figure 9. The stabilizing probability in Figure 9 is between 0.2 and 0.25 . The new introduction of the depression pills/tablets, which have a cyclic effect on the individual (in this case, we used the function $\epsilon(t)=0.167(1+\sin (t)))$ changes the stabilizing frequency to a new value between 0.1 and 0.15 which is much lower than the previous one. This shows that the effect of medication is not negligible. Together with the improvised technique of consultation, this medication is also effective in bringing down the probability of depression.

3.7. Effect of Subsequent Visits to Doctor. In this section, we will like to take a quick look at the effect of mere visits to doctor on the depression. The following differential equation will explain the situation. Let an individual visit the doctor $N$ number of times. Each time he/she visits, we assume the probability of improvement is increased by a small extent. Let the effect after the first visit be $\rho$, let the second be $\rho+\rho b$, and, after the $N$ th visit, let it be $\rho+\rho b+\cdots+\rho b^{N-1}=\rho\left(\left(1-b^{N}\right) /(1-\right.$ $b)$ ). We will choose $\rho$ and $b$ such that $\rho /(1-b)<1$. This leads to the following differential equation:

$$
p_{1}^{\prime}=-\left(\rho \frac{1-b^{N}}{1-b}+r(t)\right) p_{1}+r(t) .
$$

We will explore this special case graphically. Figure 12 of this ODE has been obtained using the parameter values, $r(t)=0.079(1+\sin (t)), b=0.167, \rho=0.236$.

From Figure 12, it is clear that increasing the number of visits to the doctor does not affect the level of depression if the rate of treatment and the factors causing depression stay constant.

\section{Conclusion}

The paper has successfully discussed different aspects of treatment of medical depression through the construction of a two-state Markov chain model. It is observed that improving the treatment strategy, which either involves providing better techniques of consultation every time a patient visits a doctor or even increasing the contact hours with the patient, would result in boosting the patients' self-confidence and decrease the rate of depression significantly. The medications available in the market are also effective in controlling the depression at the extreme stage. The drugs may lower the probability of the depressed state temporarily, but as the effect in the brain decays, the rate of moving towards a depressed state increases. But since these effective strategies do not last forever, the rate of depression increases as the treatment strategy is stopped. It would not reach the same level as before, but it would 

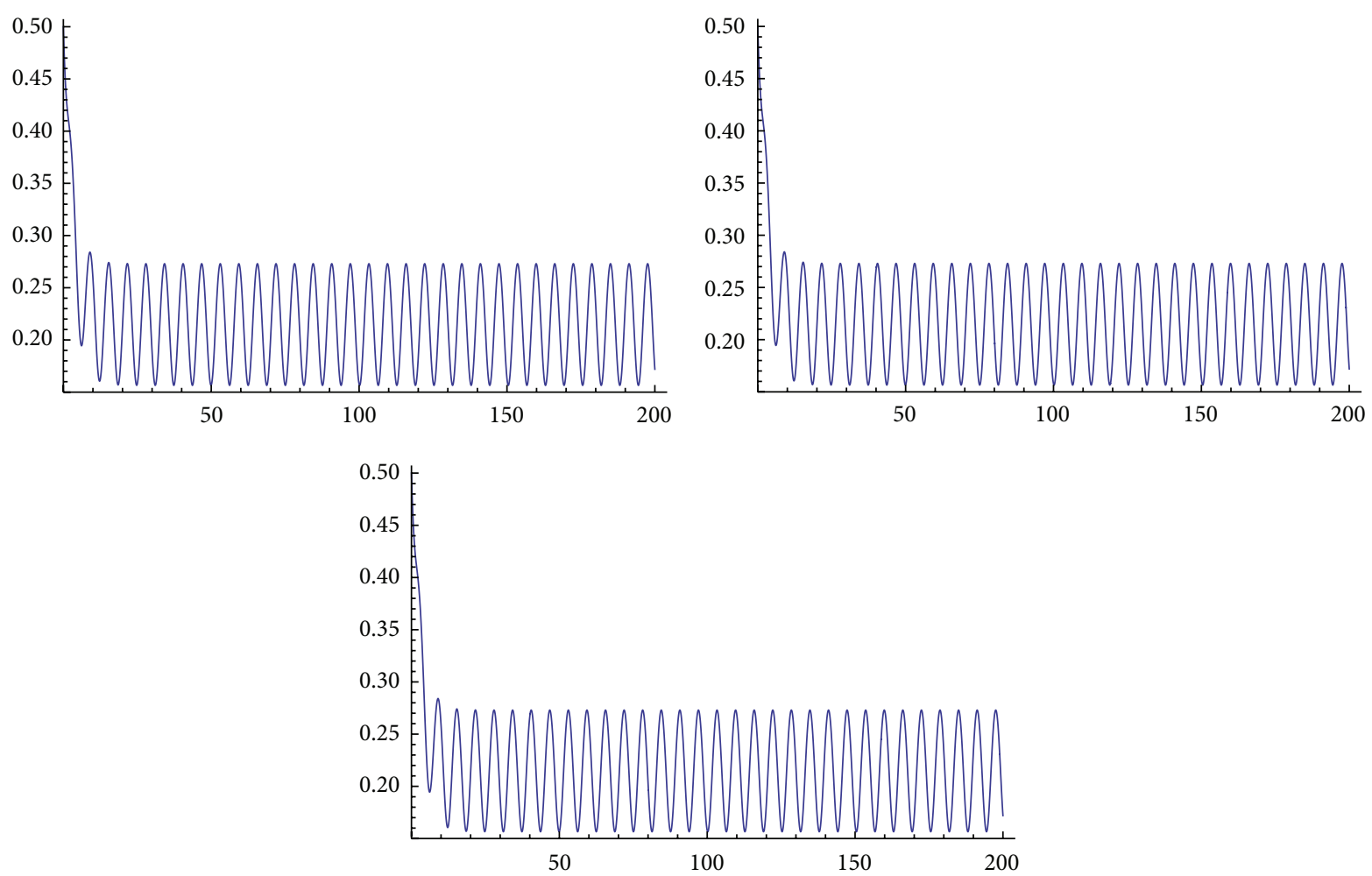

FIGURE 12: Behaviour of $p_{1}$ in the presence of treatment through consultation at different values of $N=10,50,70$, respectively.

rather take some inner force that should have been developed within an individual after the treatment phase, to drive out the depression from the patient's life. The medical treatment and consultations are effective in developing the inner strength which would serve as a shield to protect the respective individual from external forces.

\section{Conflict of Interests}

The author declares that there is no conflict of interests regarding the publication of this paper.

\section{References}

[1] S. K. Oskooyee, A. M. Rahmani, and M. R. M. Kashani, "Predicting the severity of major depression disorder with the Markov chain model," in Proceedings of the International Conference on Bioscience, Biochemistery and Bioinformatics, vol. 5, Singapoure, 2011.

[2] R. J. Davidson, D. A. Lewis, L. B. Alloy et al., "Neural and behavioral substrates of mood and mood regulation," Biological Psychiatry, vol. 52, no. 6, pp. 478-502, 2002.

[3] B. Fiemke, M. Hoogendoorn, C. A. Klein, and J. Treur, "Modeling the dynamics of mood and depression," in Proceedings of the 18th European Conference on Artificial Intelligence (ECAI '08), pp. 266-270, 2008.

[4] J. Best, M. Reed, and H. F. Nijhout, "Models of dopaminergic and serotonergic signaling," Pharmacopsychiatry, vol. 43, supplement 1, pp. S61-S66, 2010.
[5] J. Best, G. Oakley, M. Reed, and H. F. Nijhout, "Mathematical models: interactions between serotonin and dopamine in Parkinsons disease," in Etiology and Pathophysiology of Parkinson's Disease, InTech, 2011.

[6] A. B. Burrows, J. N. Morris, S. E. Simon, J. P. Hirdes, and C. Phillips, "Development of a minimum data set-based depression rating scale for use in nursing homes," Age and Ageing, vol. 29, no. 2, pp. 165-172, 2000.

[7] H. C. Tuckwell and R. M. Miura, "A mathematical model for spreading cortical depression," Biophysical Journal, vol. 23, no. 2, pp. 257-275, 1978.

[8] P. F. Lovibond and S. H. Lovibond, "The structure of negative emotional states: comparison of the Depression Anxiety Stress Scales (DASS) with the beck depression and anxiety inventories," Behaviour Research and Therapy, vol. 33, no. 3, pp. 335343, 1995.

[9] T. A. Brown, B. F. Chorpita, W. Korotitsch, and D. H. Barlow, "Psychometric properties of the Depression Anxiety Stress Scales (DASS) in clinical samples," Behaviour Research and Therapy, vol. 35, no. 1, pp. 79-89, 1997.

[10] J. D. Henry and J. R. Crawford, "The short-form version of the Depression Anxiety Stress Scales (DASS-21): construct validity and normative data in a large non-clinical sample," British Journal of Clinical Psychology, vol. 44, no. 2, pp. 227-239, 2005.

[11] J. F. Cohn, T. S. Kruez, I. Matthews et al., "Detecting depression from facial actions and vocal prosody," in Proceedings of the IEEE 3rd International Conference on Affective Computing and Intelligent Interaction and Workshops (ACII '09), September 2009.

[12] P. Ekman and E. Rosenberg, What the Face Reveals: Basic and Applied Studies of Spontaneous Expression Using the Facial 
Action Coding System (FACS), Oxford University Press, New York, NY, USA, 1997.

[13] C. Pearce, K. Dwan, M. Arnold, C. Phillips, and S. Trumble, "Doctor, patient and computer-a framework for the new consultation," International Journal of Medical Informatics, vol. 78, no. 1, pp. 32-38, 2009.

[14] A. A. Aziz, M. C. A. Klein, and J. Treur, "Modeling an ambient agent to support depression relapse prevention," in Proceedings of the IEEE/WIC/ACM International Conference on Web Intelligence and Intelligent Agent Technology-Workshops (WI-IAT '09), vol. 3, pp. 335-340, IEEE Computer Society, September 2009.

[15] W. Jizheng, H. Bin, P. Moore, and R. Ashford, "Intelligent mobile computing to assist in the treatment of depression," in Proceedings of the 3rd International Conference on Pervasive Computing and Applications (ICPCA '08), pp. 650-655, October 2008.

[16] F. G. Lether, "Shifted rectangular quadrature rule approximations to Dawson's integral $F(x)$," Journal of Computational and Applied Mathematics, vol. 92, no. 2, pp. 97-102, 1998.

[17] M. Bostani, "Almost periodic solutions for first-order differential equations," Differential and Integral Equations, vol. 19, no. 1, pp. 91-120, 2006. 

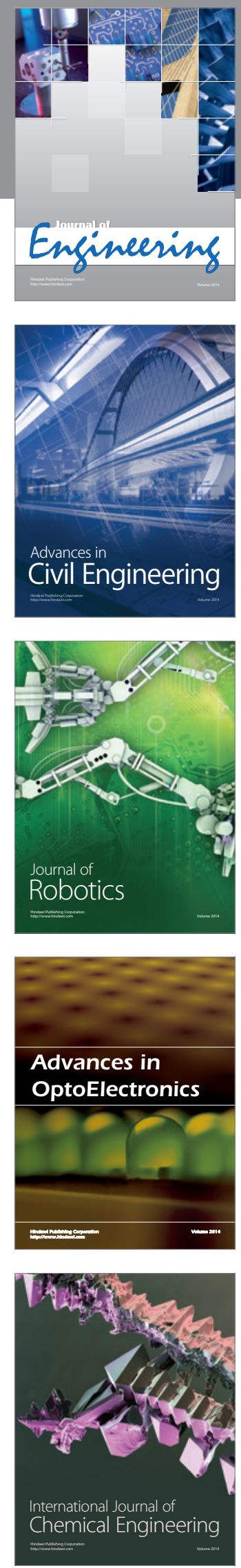

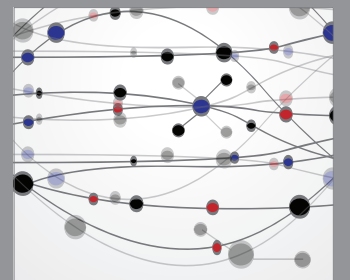

The Scientific World Journal
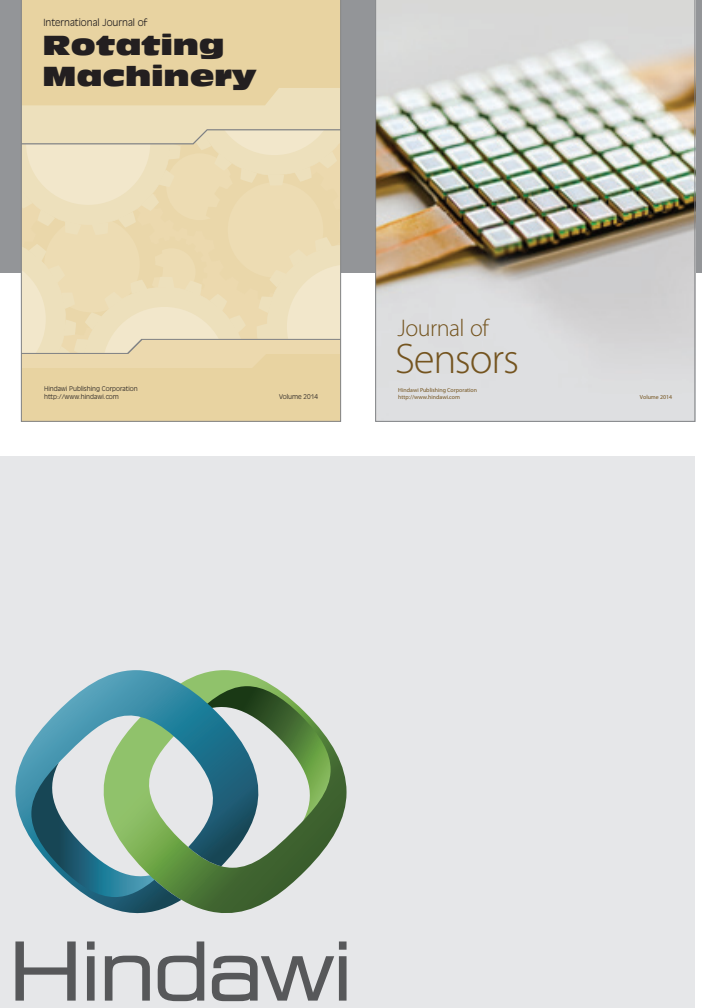

Submit your manuscripts at http://www.hindawi.com
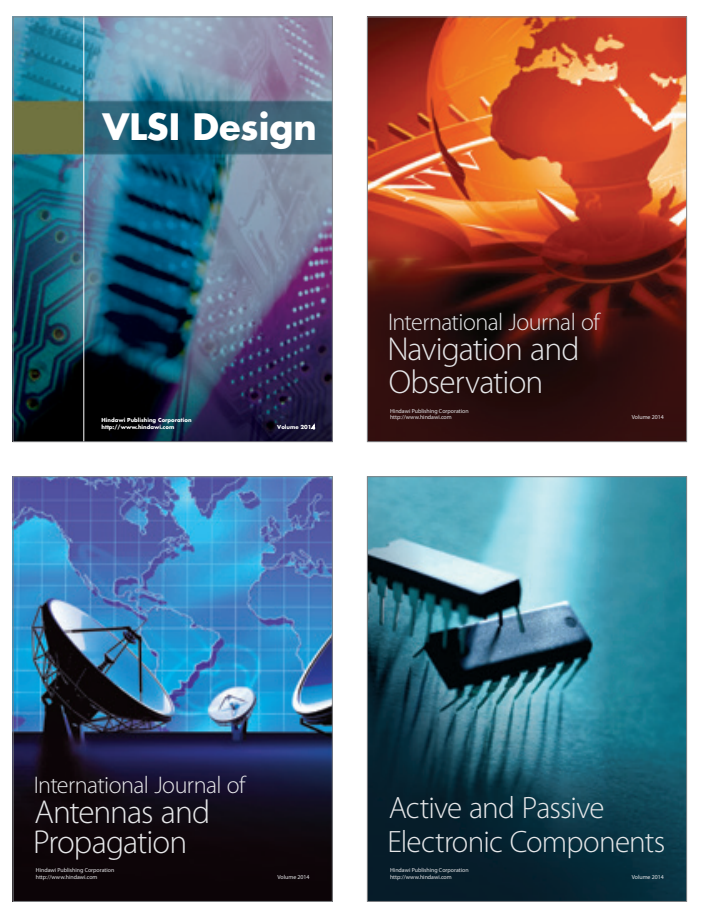
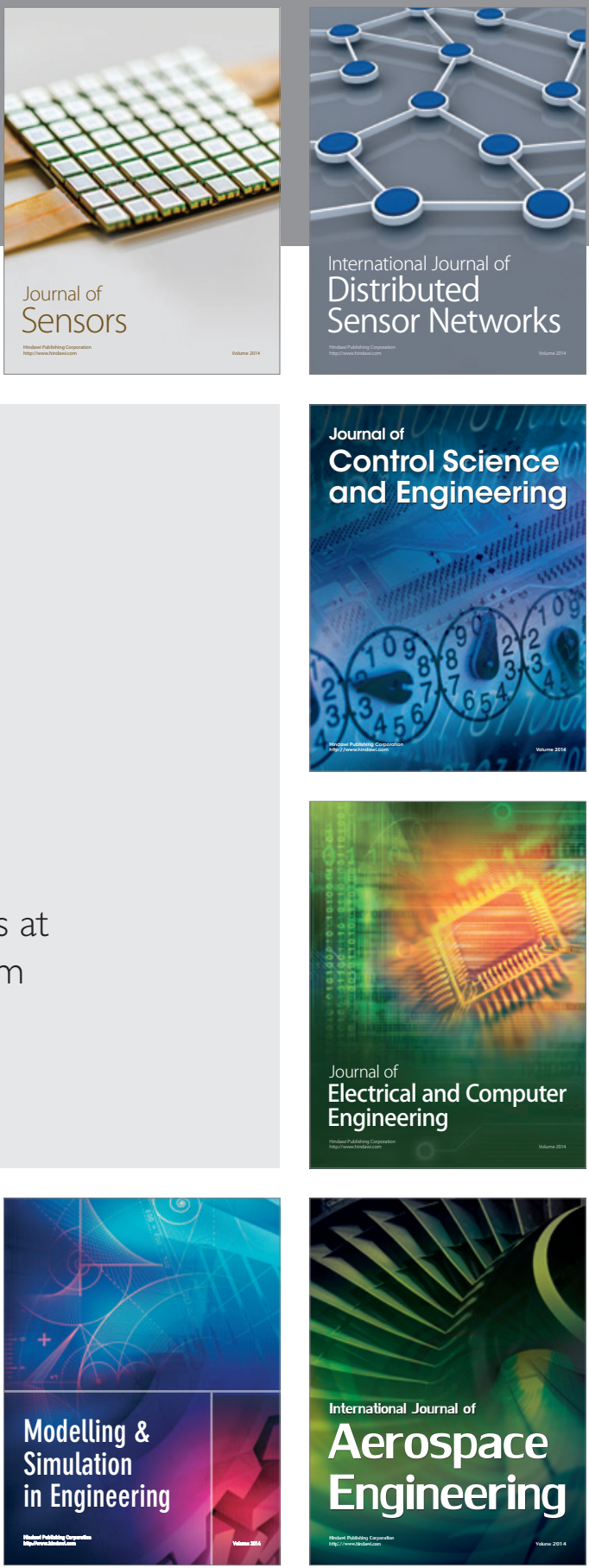

Journal of

Control Science

and Engineering
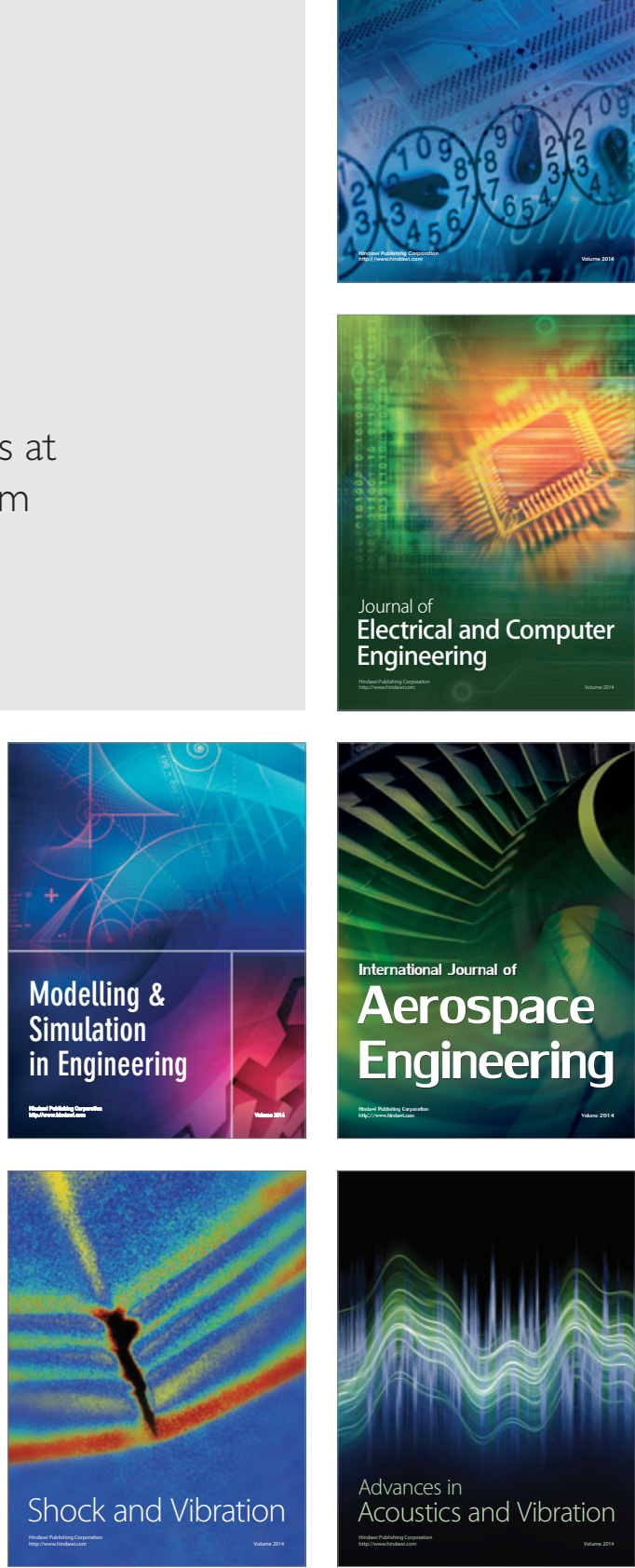